\title{
DETECCIÓN DEL VIRUS DEL PAPILOMA HUMANO EN MUESTRAS OBTENIDAS MEDIANTE TÉCNICA DE AUTOTOMA EN UN GRUPO DE UNIVERSITARIAS PERUANAS
}

\author{
Javier Manrique-Hinojosa ${ }^{1, a}$, María del Carmen Núñez-Teran ${ }^{1, b}$, Lizetth Pretel-Ydrogo ${ }^{1, b}$, \\ Yasser Sullcahuaman-Allende ${ }^{1, c}$, Ysis Roa-Meggo, ${ }^{2, \mathrm{~d}}$, Patricia Juárez-Coello ${ }^{2, \mathrm{e}}$, Stephanie Navarro-Egúsquiza ${ }^{2, e}$
}

\begin{abstract}
RESUMEN
Estudio transversal que determinó la frecuencia y genotipos del virus del papiloma humano de alto riesgo (VPH-AR) a través de la técnica de autotoma en un grupo de universitarias de Lima. Participaron 221 estudiantes y se detectó el ADN del VPH-AR con el método de reacción en cadena de la polimerasa (PCR). La frecuencia del VPH-AR en las participantes fue de 43,4\%; de este grupo se encontraron los genotipos VPH 16 en el 15,6\% y VPH 18 en el 4,2\% y otros VPH-AR en el $80,2 \%$. Se concluye que la frecuencia del VPH-AR es mayor en el grupo de universitarias de este estudio en comparación a investigaciones nacionales previas.
\end{abstract}

Palabras clave: Infecciones por Papillomavirus; Pruebas de ADN del Papillomavirus Humano; Tamizaje Masivo; Estudiantes (fuente: DeCS BIREME).

\section{DETECTION OF THE HUMAN PAPILLOMAVIRUS IN SAMPLES OBTAINED BY SELF-COLLECTION TECHNIQUE IN A GROUP OF PERUVIAN COLLEGE STUDENTS}

\begin{abstract}
Cross-sectional study that determined the frequency and the genotypes of the (HR-HPV) high-risk human papillomavirus through the self-collection technique in a group of college students of Lima. Two hundred twenty-one (221) students participated and the DNA of the HR-HPV was detected with polymerase chain reaction (PCR). The frequency of HR-HPV in participants was $43.4 \%$; of this group, genotype HPV 16 was present in $15.6 \%$, HPV 18 in $4.2 \%$, and another HR-HPV in $80.2 \%$. We can conclude that the frequency of HR-HPV is greater in the group of college students of this study in comparison with previous national investigations.
\end{abstract}

Keywords: Papillomavirus Infections; Human Papillomavirus DNA Tests; Mass Screening; Students (source: MeSH NLM).

\section{INTRODUCCIÓN}

El cáncer de cuello uterino es un problema de salud pública que amenaza la vida de las mujeres. Según GLOBOCAN en el 2018, se presentaron 569847 casos nuevos y 311365 mujeres fallecieron por esta causa a nivel mundial ${ }^{(1)}$. En el Perú, el Instituto Nacional de Enfermedades Neoplásicas (INEN) reportó 1632 de casos nuevos de cáncer de cuello uterino durante el 2016, siendo la neoplasia más frecuente seguida del cáncer de mama ${ }^{(2)}$.

La infección por el virus del papiloma humano de alto riesgo (VPH-AR) y la respuesta inmune que condiciona la persistencia de estos genotipos, se relacionan con el desarrollo del cáncer de cuello uterino ${ }^{(3)}$. Además, el consumo del tabaco, la edad del primer embarazo, la multiparidad, el uso de anticonceptivos orales y múltiples parejas sexuales son factores de riesgo asociados con la progresión de la infección crónica a lesiones premalignas ${ }^{(4)}$.

La prevalencia del VPH-AR en mujeres jóvenes es variable. En un estudio de universitarias brasileñas, se obtuvo una prevalencia de $9,8 \%$ y se identificaron principalmente los

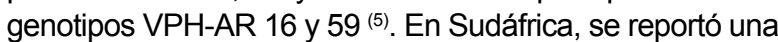
prevalencia de $54,5 \%$ en mujeres menores de 31 años que asistieron a los servicios de atención primaria ${ }^{\left({ }^{6}\right)}$. A nivel

\footnotetext{
Instituto Nacional de Enfermedades Neoplásicas. Lima, Perú.

Universidad San Martín de Porres. Lima, Perú.

a Médico especialista en Cirugía General y Oncológica; ${ }^{b}$ obstetra especialista en Atención Primaria con mención en Cáncer Ginecológico; ${ }^{\mathrm{c}}$ médico genetista; biólogo molecular; ${ }^{\mathrm{d}}$ doctora en Educación; ${ }^{\mathrm{e}}$ obstetra.

Recibido: 06/02/2018 Aprobado: 14/11/2018 En línea: 21/12/2018
}

Citar como: Manrique-Hinojosa J, Núñez-Teran M, Pretel-Ydrogo L, Sullcahuaman-Allende Y, Roa-Meggo Y, Juárez-Coello P, Navarro-Egúsquiza S. Detección del virus del papiloma humano en muestras obtenidas mediante técnica de autotoma en un grupo de universitarias peruanas. Rev Peru Med Exp Salud Publica. 2018;35(4):642-6. doi:10.17843/rpmesp.2018.354.3450. 
nacional, son escasos los estudios sobre prevalencia por el VPH-AR en mujeres jóvenes; así, un estudio en Lima encontró que el $7,7 \%$ de 321 estudiantes de nivel superior presentó la infección del VPH de los genotipos 16 y $18{ }^{(7)}$.

Para la detección del VPH con pruebas moleculares, se utiliza la autotoma, como una estrategia para incrementar la cobertura del tamizaje. Si bien la sensibilidad y especificidad de la prueba del VPH con muestras vaginales colectadas por autotoma es menor en comparación con las muestras cervicales tomadas por el personal de salud, sus valores son aceptables ${ }^{\left({ }^{8}\right)}$. En el Perú, se estudió la preferencia y satisfacción de 97 usuarias respecto a un programa de tamizaje con pruebas que utilizan la autotoma para la detección del VPH, obteniendo que un $68,8 \%$ prefirieron esta alternativa, por la menor demanda de tiempo y mayor privacidad como las principales razones de preferencia ${ }^{(9)}$. No obstante, la inseguridad que la prueba se realice correctamente fue el motivo para que las mujeres opten por la asistencia del personal de salud.

El objetivo de la investigación fue determinar la frecuencia y genotipos del VPH-AR a través de la técnica de autotoma en un grupo de universitarias de Lima.

\section{EL ESTUDIO}

Estudio transversal, realizado en la Facultad de Obstetricia y Enfermería de la Universidad de San Martín de Porres, institución educativa privada de Lima Metropolitana, durante el periodo de marzo a noviembre de 2016. Se invitó a las estudiantes a participar en la investigación, tomando en cuenta que durante su formación académica recibieron previamente información sobre los tipos de tamizaje para el cáncer de cuello uterino, por lo cual, se esperó menor rechazo a la técnica de autotoma.

Se incluyó a través de una selección no probabilística a 221 estudiantes, quienes participaron voluntariamente y cumplieron con los siguientes criterios de inclusión: ser sexualmente activas, tener entre 18 a 30 años, estar aptas para la prueba (no haber tenido relaciones sexuales ni aplicado óvulos o cremas vaginales 48 horas antes), y no tener conocimiento de lesiones premalignas.

Un equipo de investigadores dirigidos por médicos oncólogos y obstetras especialistas en atención primaria con mención en cáncer ginecológico del INEN, explicaron los objetivos del estudio y capacitaron a las estudiantes en relación a la técnica de la autotoma vaginal para la prueba de detección de VPH, según la «Guía para toma de la muestra de pruebas moleculares de detección del VPH» del INEN. La capacitación se realizó mediante dos sesiones demostrativas de tres horas en grupos de 15 alumnas y se emplearon maquetas anatómicas y kits para la autotoma.

\section{MENSAJES CLAVE}

Motivación para realizar el estudio. Conocer la frecuencia de la infección y la distribución de los genotipos del virus del papiloma humano de alto riesgo (VPH-AR) a través de la detección con pruebas moleculares que utilizan la técnica de autotoma en un grupo de universitarias de Lima.

Principales hallazgos. Se encontró una alta frecuencia del VPH-AR $(43,4 \%)$ en comparación con estudios nacionales previos; además se hallaron los genotipos VPH 16 en el 15,6\%, VPH 18 en el 4,2\% y otros VPH-AR en el $80,2 \%$.

Implicancias. Se requiere potenciar las actividades de educación en las jóvenes sobre la infección por el VPH-AR.

Se elaboró un cuestionario anónimo de seis preguntas cerradas para obtener información respecto las variables: edad, inicio de actividad sexual, número de parejas sexuales, uso de métodos anticonceptivos y fecha de la prueba de Papanicolaou. El cuestionario fue validado por juicio de expertos, los cuales fueron especialistas en oncología del INEN.

Se entregó para la colección de la muestra vaginal el Abbott Real Time Detection VPH Kit a las estudiantes, que contenía un citocepillo Gynemed, el cual debía ser insertado en el canal vaginal, girándose tres veces y un tubo colector con $9 \mathrm{ml}$ de tampón de transporte de muestra (tiocianato de guanidina en tampón Tris), donde luego se lavaría el citocepillo y se dejaría el material genético en el tubo. La autotoma de la muestra vaginal se realizó en el Centro Especializado de Salud Sexual y Reproductiva de Adolescentes (CESSRA) de la facultad, luego entregaron el tubo colector y llenaron el cuestionario.

Las muestras fueron procesadas en el Laboratorio del Equipo Funcional de Genética y Biología Molecular del INEN. La prueba permite detectar 14 genotipos del VPH-AR $(16,18,31,33,35,39,45,51,52,56,58,59,66$ y 68). Esta prueba reportó de manera separada los genotipos 16 y 18 . El equipo estuvo compuesto por una unidad m2000sp que se encargó de preparar el ácido nucleico y un analizador m2000rt que realizó la PCR en tiempo real. Se utilizó una mezcla de múltiples primers, y sondas para la amplificación y detección de ADN del VPH-AR. Como control de calidad de las células cervicales, recogidas en citología en medio líquido, se utilizó el gen de $\beta$ globina ${ }^{(8)}$.

El análisis se realizó con el programa SPSS versión 21 (SPSS, Inc., Chicago, IL, EE. UU.). La parte descriptiva incluyó frecuencias absolutas y relativas para las variables cualitativas y medidas de tendencia central para variables cuantitativas. El análisis bivariado se realizó mediante la prueba de chi cuadrado para evaluar la asociación entre la infección por el VPH-AR y las variables: edad ( $\leq 22$ años 
y 22 a 30 años), inicio de las relaciones sexuales ( $\leq 17$ años; 18 a más años), número de parejas sexuales ( $\leq 2$; tres a más), y uso de anticonceptivos.

El estudio fue aprobado por el Comité de Ética e Investigación de la Facultad de Obstetricia y Enfermería de la Universidad de San Martin de Porres. Se administró y obtuvo el consentimiento informado de todas las participantes.

\section{RESULTADOS}

Se incluyeron un total de 221 universitarias; respecto a la edad, la media fue de 22,4 años. El promedio de inicio de las relaciones sexuales fue de 17,6 años y una mediana de dos parejas sexuales. El 18,9\% no utilizaba métodos anticonceptivos y del grupo que sí usaba anticonceptivos, el preservativo fue el de mayor frecuencia (28,4\%). El 62,9\% se realizó la prueba de Papanicolaou durante los tres años previos al estudio, mientras que el $22,2 \%$ nunca se hizo la prueba (Tabla 1).

La frecuencia del VPH-AR fue de 43,4 \%, en relación a la distribución de los genotipos, en el grupo que presentó VPH-AR, se identificó el genotipo 16 en el $15,6 \%$, el 18 en el $4,2 \%$, y el grupo de otros genotipos del VPH-AR $(31,33$, $35,39,45,51,52,56,58,59,66$ y 68 ) en el $80,2 \%$. Se encontró una infección simple por VPH 16 en el 2,1\% y por el VPH 18 en el 2,1\%. Asimismo, se halló la infección mixta por el VPH 16 más el grupo de otros genotipos del VPHAR en el $13,9 \%$ y por el VPH 18 más el grupo de otros genotipos del VPH-AR en el 2,1\% (Tabla 2).

Tabla 1. Características de estudiantes universitarias de universidad privada de Lima Metropolitana $(n=221)$

\begin{tabular}{lc}
\hline Características & $\mathbf{n}(\%)$ \\
\hline Edad (DE) & $22,4(2,9)$ \\
\hline Inicio de relaciones sexuales (DE) & $17,6(2,2)$ \\
Número de parejas sexuales, mediana (RIC) & $2(1-11)$ \\
\hline Métodos Anticonceptivos & \\
$\quad$ No usa & $28(18,9)$ \\
$\quad$ Ritmo & $3(1,4)$ \\
$\quad$ Preservativo & $42(28,4)$ \\
$\quad$ Píldoras anticonceptivas & $27(12,2)$ \\
$\quad$ Inyectable & $38(17,2)$ \\
$\quad$ Implante hormonal & $10(4,5)$ \\
Último Papanicolaou & \\
$\quad$ Nunca & $49(22,2)$ \\
$\quad \leq 3$ años anteriores al estudio & $95(62,9)$ \\
$\quad \geq 4$ años anteriores al estudio & $7(3,2)$ \\
\hline
\end{tabular}

DE: desviación estándar; RIC: rango intercuartílico
Tabla 2. Frecuencia de infección por el virus del papiloma humano de alto riesgo en estudiantes universitarias y distribución de los genotipos

\begin{tabular}{|c|c|}
\hline Tipo de infección & n (\%) \\
\hline \multicolumn{2}{|c|}{ Virus del papiloma humano de alto riesgo $(n=221)$} \\
\hline Positivo & $96(43,4)$ \\
\hline Negativo & $125(56,6)$ \\
\hline \multicolumn{2}{|c|}{ Distribución de genotipos ( $\mathrm{n}=96)$} \\
\hline \multicolumn{2}{|l|}{ Genotipos simples } \\
\hline VPH 18 & $2(2,1)$ \\
\hline VPH 16 & $2(2,1)$ \\
\hline \multicolumn{2}{|l|}{ Genotipos mixtos } \\
\hline VPH 16/Otros VPH-AR & $13(13,5)$ \\
\hline VPH 18/Otros VPH-AR & $2(2,1)$ \\
\hline Otros VPH-AR & $77(80,2)$ \\
\hline
\end{tabular}

VPH: virus del papiloma humano; AR: alto riesgo

En relación con las características estudiadas, se encontró que los intervalos de edad ( $\leq 22$ años y 22 a 30 años), la edad de inicio de las relaciones sexuales y el uso de anticonceptivos no presentaron significancia estadística. Sin embargo, el número de parejas sexuales sí se relacionó con el resultado negativo y positivo para el VPH-AR $(p=0,003)$. De los casos positivos para VPH-AR, el $54,9 \%$ indicaron tener hasta dos parejas sexuales y el $45.1 \%$ de tres a más, mientras que las participantes con resultado negativo, el $74,8 \%$ señalaron tener hasta dos parejas sexuales y el $25,2 \%$ de tres a más (Tabla 3 ).

\section{DISCUSIÓN}

El estudio determinó una frecuencia de 43,4\% del VPH-AR en el grupo de universitarias con la técnica de autotoma. Esta cifra es mayor en comparación con investigaciones locales en mujeres jóvenes donde hallaron valores de 7,7\% y $18,5 \%{ }^{(7,10)}$. Incluso también es mayor a los resultados obtenidos en estudios nacionales de mujeres de distintos rangos etarios, donde la infección por $\mathrm{VPH}$ se detectó en el $34,5 \%$ y $15,6 \%{ }^{(11,12)}$. A nivel internacional, se evidenció prevalencias de $70,3 \%$ en Jamaica, $54,5 \%$ en Sudáfrica y $51,4 \%$ en Uganda, respectivamente $(6,13,14)$. Por el contrario, en países como la India y Corea del Sur, se encontraron valores de $19,2 \%$ y $9,4 \% \quad(15,16)$. Es probable que, las diferencias con algunos estudios, puedan ser explicadas parcialmente por la influencia de los determinantes sociales de la infección por el VPH y las desigualdades en salud.

El grupo de otros genotipos del VPH-AR fue el más identificado $(80,2 \%)$, a diferencia del VPH 16 y VPH 18 reportados en el $15,6 \%$ y $4,2 \%$ de las universitarias. En el país, estos datos concuerdan, con el estudio de Iwasaki et al. (11), donde el conjunto de los otros genotipos del VPH-AR obtuvo mayor 
Tabla 3. Resultados de la prueba de detección del virus del papiloma humano de alto riesgo según las características de las jóvenes universitarias.

\begin{tabular}{|c|c|c|c|}
\hline \multirow{2}{*}{ Características } & \multicolumn{2}{|c|}{ Virus del papiloma humano de alto riesgo } & \multirow{2}{*}{ Valor de $\mathrm{p}^{*}$} \\
\hline & Positivo, n (\%) & Negativo, n (\%) & \\
\hline \multicolumn{4}{|l|}{ Edad (años) } \\
\hline$\leq 21$ & $46(47,9)$ & $57(45,6)$ & 0,732 \\
\hline $22-30$ & $50(52,1)$ & $68(54,4)$ & \\
\hline \multicolumn{4}{|c|}{ Inicio de relaciones sexuales (años) } \\
\hline$\leq 17$ & $55(50,9)$ & $53(49,1)$ & 0,056 \\
\hline 18 a más & $39(37,9)$ & $64(62,1)$ & \\
\hline \multicolumn{4}{|c|}{ Número de compañeros sexuales } \\
\hline$\leq$ Dos & $50(54,9)$ & $89(74,8)$ & 0,003 \\
\hline Tres a más & $41(45,1)$ & $30(25,2)$ & \\
\hline \multicolumn{4}{|c|}{ Uso de anticonceptivos } \\
\hline No & $15(22,7)$ & $13(15,9)$ & 0,289 \\
\hline Sí & $51(77,3)$ & $69(84,1)$ & \\
\hline
\end{tabular}

* Prueba de Chi cuadrado

porcentaje (81,4\%); por el contrario, Silva et al. ${ }^{(17)}$ indicaron que el VPH 16 fue el más detectado (38,5\%). La literatura internacional sobre distribución de los genotipos del VPH en mujeres con citología normal señala que, a nivel individual, el genotipo 16 es el más frecuente. Sin embargo, cuando se coteja con el colectivo de otros VPH-AR, este muestra mayor prevalencia. Es así que la diferencia entre el VPH 16 y el grupo de otros genotipos del VPH-AR obtenida en el estudio es similar a las encontradas por autores como Chen et al. ${ }^{(18)}$ (17,6\% vs. $77,6 \%$ ) y Leinonen et al. ${ }^{(19)}(14,5 \%$ vs. $68,5 \%)$.

Respecto a las características de las universitarias incluidas, como edad, inicio de actividad sexual, uso de anticonceptivos y número de parejas sexuales, sólo esta última característica presentó una asociación significativa en el estudio. De forma similar el trabajo de Silva et al. ${ }^{(17)}$, indicó que el porcentaje del VPH fue superior en mujeres que reportaron tener más de tres parejas sexuales. Valderrama et al. ${ }^{(7)}$ señalaron que a mayor diferencia de edad con la pareja sexual masculina, se incrementa el riesgo de infección por el VPH. De acuerdo con los estudios internacionales en mujeres jóvenes de las regiones con alta prevalencia del VPH-AR, el comportamiento sexual se evidencia como en el principal factor de riesgo.
Las variables que indicaron mayor probabilidad de infección por VPH, fueron inicio de la actividad sexual (13), número de parejas sexuales ${ }^{(14)}$ y no usar preservativo ${ }^{(20)}$.

Dentro de las limitaciones del estudio, se encuentra el tipo de muestreo no probabilístico que se realizó, el cual impide extrapolar los resultados a otras poblaciones. Además, es posible que las universitarias decidieran su participación, por tener mayor exposición a factores de riesgo de la infección por el VPH.

En conclusión, la frecuencia del VPH-AR es alta en el grupo de universitarias de este estudio en comparación a investigaciones nacionales previas. En tal sentido, es indispensable promover comportamientos sexuales saludables, educación sobre la relación del VPH-AR y el cáncer de cuello uterino, y un futuro tamizaje oportuno en esta población de riesgo.

Fuentes de financiamiento: La presente investigación fue financiada por el laboratorio Abbott, el cual proporcionó los kits para la obtención de las muestras vaginales.

Conflicto de intereses: Los autores declaran no tener ningún conflicto de interés.

\section{REFERENCIAS BIBLIOGRÁFICAS}

1. GLOBOCAN. Cervix uterine [Internet]. Lyon, France: World Health Organization; 2018 [citado el 01 de octubre del 2018]. Disponible en: http://gco.iarc.fr/ today/data/factsheets/cancers/23-CervixUteri-fact-sheet.pdf

2. Instituto Nacional de Enfermedades Neoplásicas. Casos nuevos de cáncer registrados en INEN periodo 2000-2016 [Internet]. INEN; 2016 [citado el 01 de octubre del 2018]. Disponible en: https://portal.inen. sld.pe/wp-content/uploads/2018/06/ INEN-CASOS-NUEVOS-2000-2016. pdf

3. Stensen $S$, Kjaer $S$, Jensen $S$, Frederiksen $\mathrm{K}$, Junge J, Iftner $\mathrm{T}$, et al. Factors asso- ciated with type-specific persistence of high-risk human papillomavirus infection: A population-based study. Int J Cancer. 2016;138(2):361-8. doi: 10.1002/ ijc.29719.

4. Momenimovahed Z, Salehiniya H. Cervical cancer in Iran: integrative insights of epidemiological analysis. Biomedicine 
(Taipei). 2018;8(3):18. doi: 10.1051/bm$\mathrm{dcn} / 2018080318$.

5. Covre R, Valente J, Primo E, Mendonça M, Yamamoto M, Yassui E, et al. Prevalence of type-specific HPV among female university students from northern Brazil. Infect Agent Cancer. 2015;10:21. doi: 10.1186/s13027-015-0017-x.

6. Ebrahim S, Mndende XK, Kharsany AB, Mbulawa ZZ, Naranbhai V, Frohlich J, et al. High Burden of Human Papillomavirus (HPV) Infection among Young Women in KwaZulu-Natal, South Africa. PLoS One. 2016;11(1):e0146603. doi: 10.1371/journal.pone.0146603.

7. Valderrama M, Campos F, Cárcamo C, García P. Factores asociados a lesiones cervicales o presencia del virus del papiloma humano en dos poblaciones de estudiantes en Lima. Rev Peru Med Exp Salud Publica. 2007;24(3):234-39. doi: http://dx.doi. org/10.17843/rpmesp.2007.243.1112.

8. Organización Panamericana de la Salud. Incorporación de la Prueba del Virus del Papiloma Humano en Programas de Prevención de Cáncer Cervicouterino. Washington DC: OPS; 2016.

9. Morán $\mathrm{F}$, Cárcamo C, Valderrama $\mathrm{M}$, García PJ. Preferencias y satisfacción hacia un programa de tamizaje con pruebas autoadministradas de deteccion del virus de papiloma humano. Rev Peru Med Exp Salud Publica. 2017;34(2):228-32. doi: 10.17843/rpmesp.2017.342.2453.

10. Sullcahuaman-Allende Y, Castro-Mujica M, Mejía R, Castañeda C, Castillo M, Dolores-Cerna K, Poquioma E. Características sociodemográficas de mujeres peruanas con Virus del Papiloma Humanos detectado por PCR-RFLP. Rev Peru Med Exp Salud Publica. 2015;32(3):509-14. doi: http://dx.doi.org/10.17843/rpmesp.2015.323.1684.

11. Iwasaki R, Gálvez-Philpott F, Arias-Stella J. Jr, Arias-Stella J. Prevalence of high-risk human papillomavirus by cobas 4800 HPV test in urban Peru. Braz J Infect Dis. 2014;18(5):469-72. doi: 10.1016/j. bjid.2014.01.010.

12. Manrique J, Sarria G, Arias A, Mora P, Limache A, Núñez M, et al. HPV detection by PCR in real time in Peruvian women. Cancer Res. 2014;74(19 Suppl):3488. doi:10.1158/1538-7445.AM2014-3488.

13. Watt A, Ragin C, Younger N, Garwood D, Jackson M, Smikle M, et al. High-risk human papillomavirus (HPV) genotypes in Jamaican women. Cancer Res. 2007;67(9):LB122.

14. Banura C, Franceschi S, Doorn L, Arslan A, Wabwire-Mangen F, Mbidde E, et al. Infection with Human Papillomavirus and HIV among Young Women in Kampala, Uganda. J Infect Dis. 2008;197(4):55562. doi: $10.1086 / 526792$.

15. Garg A, Suri V, Nijhawan R, Aggarwal N, Aggarwal R, Guleria C. Prevalence of Human Papilloma Virus Infection in Young Primiparous Women During Postpartum Period: Study from a Tertiary Care Center in Northern India. J Clin Diagn Res. 2016;10(10):QC06-QC09.

16. Hai S. Korea: Cervical Cancer Screening and Epidemiology. Infectious Diseases in Obstetrics and Gynecology [interent]. 2006 [citado el 22 de octubre de 2017];212. Disponible en: https://eds.b.ebscohost. $\mathrm{com} / \mathrm{ehost} / \mathrm{detail} /$ detail ?vid=0\&sid=f0f$174 \mathrm{cb}-7 \mathrm{cde}-4 \mathrm{c} 8 \mathrm{a}-\mathrm{a} 0 \mathrm{a} 2-\mathrm{a} 502 \mathrm{fb} 23 \mathrm{e} 688 \%$ 40 sessionmgr $101 \&$ bdata $=J$ mxhb -
mc9ZXMmc210ZT1laG9zdC1saX$\mathrm{Zl} \# \mathrm{AN}=287746628 \mathrm{db}=\mathrm{a} 9 \mathrm{~h}$

17. Silva-Caso W, Olivera-Irazábal M, León-Álvarez P, del Valle L, Díaz-Estacio $S$, Vargas M, et al. Identification of human papillomavirus as a preventive strategy for cervical cancer in asymptomatic women in the Peruvian Andes. Asian Pac J Trop Med. 2014;7S1:S121-6. doi: 10.1016/ S1995-7645(14)60217-0.

18. Chen X, Xu H, Xu W, Zeng W, Liu J, Wu $\mathrm{Q}$, et al. Prevalence and genotype distribution of human papillomavirus in 961,029 screening tests in southeastern China (Zhejiang Province) between 2011 and 2015. Sci Rep. 2017;7(1):14813. doi: 10.1038/s41598-017-13299-y.

19. Leinonen M, Anttila A, Malila N, Dillner J, Forslund O, Nieminen P. Type- and age-specific distribution of human papillomavirus in women attending cervical cancer screening in Finland. Br J Cancer. 2013;109(11):2941-50. doi: 10.1038/ bjc.2013.647.

20. Devarakonda S, Neppalli A, Liu L, Friday E, Shi R. Risk factors and prevalence of genital HPV infection among adult females in US between 20032010: Data from NHANES study. Cancer Res. 2014;74(19 Suppl):278. doi:10.1158/1538-7445.AM2014-278.

Correspondencia: Ysis Roa Meggo.

Dirección: Av. Salaverry 1136-1144, Jesús

Maria-Lima.

Teléfono: 4711171.

Email:yroam@usmp.pe

\section{Nuestros artículos se encuentran indizados en:}

$$
\text { PublMed }
$$

